\title{
The Ototoxicity of Chloroquine and Hydroxychloroquine: A Systematic Review
}

\author{
Carolina Pereira Fernandes ${ }^{10} \quad$ Luíza Silva Vernier $^{2}{ }^{(0)}$ \\ ${ }^{1}$ Language and Hearing Sciences at the Universidade Federal de \\ Ciências da Saúde de Porto Alegre (UFCSPA), Porto Alegre, RS, Brazil \\ 2 Graduate Program in Health Sciences at Universidade Federal de \\ Ciências da Saúde de Porto Alegre (UFCSPA), Porto Alegre, RS, Brazil \\ ${ }^{3}$ Analytical Toxicology and Graduate Program of Health Sciences at \\ Universidade Federal de Ciências da Saúde de Porto Alegre \\ (UFCSPA), Porto Alegre, RS, Brazil \\ ${ }^{4}$ Speech-Language Pathology and Audiology Program at \\ Universidade Federal de Ciências da Saúde de (UFCSPA), Porto \\ Alegre, RS, Brazil
}

Eliane Dallegrave ${ }^{30}$ Márcia Salgado Machado ${ }^{4}$

\author{
Address for correspondence Márcia Salgado Machado, PhD, Rua \\ Sarmento Leite, 245 - Centro Histórico, Porto Alegre - RS, 90050-170, \\ Brazil (e-mail: marciasm@ufcspa.edu.br).
}

Int Arch Otorhinolaryngol 2022;26(1):e167-e177.

\author{
Abstract \\ Keywords \\ - chloroquine \\ - hydroxychloroquine \\ - ototoxicity \\ - hearing loss
}

Introduction Chloroquine and hydroxychloroquine are antimalarial drugs widely used in the treatment of rheumatic diseases. With the global pandemic caused by the new coronavirus, there was an increase in the prescription of these drugs, which led to a major concern regarding their ototoxic effects.

Objectives The objective of the present study was to assess existing scientific evidence about the toxic effects of chloroquine and hydroxychloroquine on the peripheral and/or central auditory system.

Data Synthesis A systematic literature review was performed by searching the PubMed (Medline), Scopus, Web of Science, LILACS, and SciELO electronic databases, in a search of articles that fullfiled the predefined inclusion and exclusion criteria. The review was conducted in three phases and, in all of them, analyses were performed by two independent researchers. Disagreements were discussed with a third researcher until a consensus was reached. A total of 437 articles were found and 8 were included in this review. Seven of the included studies reported hearing loss in their samples and presented a diagnostic hypothesis of ototoxicity induced by chloroquine or hydroxychloroquine. The most common type of hearing loss was sensorineural, with varying laterality and degrees of severity. The most frequently used audiological test was pure tone audiometry, and only two studies assessed brainstem evoked responses.

Conclusion The scientific evidence compiled in this research showed that chloroquine and hydroxychloroquine have an ototoxic effect in the peripheral auditory system. These drugs can cause cochlear damage, including changes in the stria vascularis and lesions in sensory hair cells. received

June 21, 2021

accepted after revision

September 19, 2021
DOI https://doi.org/ 10.1055/s-0041-1740986. ISSN 1809-9777. (c) 2022. Fundação Otorrinolaringologia. All rights reserved.

This is an open access article published by Thieme under the terms of the Creative Commons Attribution-NonDerivative-NonCommercial-License, permitting copying and reproduction so long as the original work is given appropriate credit. Contents may not be used for commercial purposes, or adapted, remixed, transformed or built upon. (https://creativecommons.org/ licenses/by-nc-nd/4.0/)

Thieme Revinter Publicações Ltda., Rua do Matoso 170, Rio de Janeiro, RJ, CEP 20270-135, Brazil 


\section{Introduction}

Chloroquine (CQ) and hydroxychloroquine (HCQ) are antimalarial agents that are widely used to treat rheumatic diseases, such as rheumatoid arthritis (RA), systemic lupus erythematosus (SLE), discoid lupus erythematosus (DLE), and Sjögren syndrome (SS). ${ }^{1-8}$ The advent of the new coronavirus (SARS-CoV-2) pandemic spurred investigations into the efficacy and safety of these two drugs in combating this virus and led to an increase in their use. ${ }^{2,9,10}$ Due to the largescale issue of prescriptions, a greater concern also arose regarding the ototoxic effects of the two drugs.

Both drugs are derived from quinoline ${ }^{1,3-5,11}$ but, although they have a similar composition, HCQ has fewer toxic properties than $\mathrm{CQ}^{4,8,9}$ Regarding pharmacokinetics, $\mathrm{CQ}$ and HCQ are well absorbed orally, with good bioavailability, wide distribution, and prolonged elimination half-life (between 40-60 days). ${ }^{12}$ They also share similar adverse side effects, such as epithelial lesions, rashes and skin hyperpigmentation, retinopathy, and other visual disorders. ${ }^{1-3,5,7,8,13}$ These are caused by strong melanin binding. ${ }^{12}$ Usually, the toxicity of these drugs is monitored through periodic ophthalmological evaluations. ${ }^{1,4,8}$ However, questions regarding ototoxicity and possible hearing disorders have not been equally considered nor assessed. ${ }^{1,5,6,14}$

There are reports on the possible changes that $\mathrm{CQ}^{1,5,13,15-20}$ and $\mathrm{HCQ}^{3,6,7,11,14,21,22}$ induce in the auditory system. However, the ototoxic action of these drugs is not fully understood and, although there are some hypotheses about the mechanisms behind their effects in the inner ear (IE), $, 18,23,24$ there is still no consensus on what happens. Despite the limited evidence regarding their ototoxicity, the World Health Organization ${ }^{25}$ has identified antimalarial drugs as possible causes of hearing loss in adults, among other types of medication.

Given the inconsistencies in the information on this topic, this study aimed to analyze existing scientific evidence about the toxic effects of CQ and HCQ on the peripheral and/or central auditory system of those who take them.

\section{Review of the Literature}

This is a systematic review of the literature based on the following research questions: "Are there audiological changes in patients exposed to CQ and HCQ?"; "If so, which ones are the most common?"; "Is there any difference between the ototoxic properties of the two drugs?" These questions were structured using the Population, Intervention, Comparison, Outcome (PICO) framework :

- Population: subjects, without any age or gender restriction, who had used CQ and/or HCQ during their medical treatment;

- Intervention: taking CQ and/or HCQ during any medical treatment;

- Comparison: individuals who were not exposed to either drug;

- Outcome: normal hearing or hearing loss due to CQ or HCQ ototoxicity.
We used the Preferred Reporting Items for Systematic Reviews and Meta-Analyses (PRISMA) ${ }^{26}$ recommendations to conduct this review and registered our research on the International Prospective Register of Systematic Reviews (PROSPERO) platform, under the registration number CRD42020182698.

\section{Inclusion and Exclusion Criteria}

To select articles, we used the following inclusion criteria:

a) Original articles published in scientific journals, with free access and no restrictions regarding the date of publication or design;

b) Studies published in Portuguese, English, Spanish, or French;

c) Research involving studies in humans;

d) Articles in which hearing loss had been exclusively caused by the use of CQ or HCQ (in any of their forms), or by a combination of these drugs with other nonototoxic medication;

e) Studies presenting data concerning peripheral and/or central hearing assessments.

Studies with the following characteristics were excluded:

a) Articles in which hearing loss could have occurred due to diseases or complications of underlying diseases in the population (i.e., diseases that may usually be treated with CQ or HCQ but are reported in the literature as risks for hearing loss, such as SLE and RA), rather than an exclusive association with CQ or HCQ use;

b) Studies assessing the hearing of infants with prenatal exposure to CQ or HCQ;

c) Literature review articles;

d) Letters to the editor and summaries for events, due to the low scientific evidence in these documents.

\section{Search Strategy and Article Selection}

Descriptors were selected using Health Sciences Descriptors (DeCs) and the Medical Subject Headings (MeSH) filters, as well as the Boolean operators "OR" and "AND" in search strategies. The electronic databases used for searching were PubMed (Medline), Scopus, Web of Science, LILACS, and SciELO.

The searches of the electronic databases were performed from May $1^{\text {st }}$ to $11^{\text {th }}, 2020$, using the following strategy: (chloroquine OR hydroxychloroquine) AND (hearing loss OR hearing impairment OR hypoacuses OR hypoacusis OR loss, hearing). No search restriction filters were used. A new search was made between January $18^{\text {th }}$ and March $7^{\text {th }}, 2021$.

In the first stage of the selection strategy, the titles and abstracts of the articles from the database search were read. The articles were chosen and selected for the next phase by two blind and independent researchers. In the second phase, the selected articles were read in full by the two researchers (following the same strategy in the previous step). Finally, a secondary search for more published research was done through a new selection of articles based on the references from all the selected studies. In the first two stages, a third researcher acted as a judge, analyzing, and comparing the 
data collected by the two independent researchers. In all phases, disagreements over the selection of articles were discussed by all researchers until there was a consensus.

All articles were accessed in full through the Coordenação de Aperfeiçoamento de Pessoal de Nível Superior (CAPES)/ Ministério da Educação (MEC) portal of journals, using remote access via the federated academic community(café, in the Portuguese acronym). When access was not available through the portal, the studies were requested from other national and international university libraries, at no cost.

\section{Data Extraction}

To perform data extraction from the selected studies, a spreadsheet was prepared using the Microsoft Excel software (Microsoft Corp., Redmond, WA, USA). The following data were extracted:

a) Basic information about the study (title, authors, year of publication, journal, language, country of origin, design, and objectives of the research);

b) Data regarding the sample (size, average age, gender, pathology in the population, and inclusion and exclusion criteria);

c) Data concerning the drugs of interest in this review (the drugs used and their dosages, forms of CQ and HCQ use, duration of treatment with these drugs, and adverse effects associated with their administration);

d) Auditory symptoms and audiological testing (hearing loss and the duration of audiological complaints, audiological tests and descriptions of these tests, hypotheses regarding the cause of hearing loss);

e) Strategies used in an attempt to reverse hearing loss;

f) Results and conclusions (results of audiological testing, characteristics of hearing loss [type, degree, and laterality], results of attempts to reverse hearing loss, results of other tests and conclusions).

\section{Data Analysis}

The studies were analyzed according to the Strengthening the Reporting of Observational Studies in Epidemiology (STROBE) initiative guidelines ${ }^{27}$ and the Grading of Recommendations, Assessment, Development, and Evaluation (GRADE) system. ${ }^{28}$ The STROBE initiative is a checklist with 22 items that represent all the information that must be contained in an article. This checklist can be used to verify that all items are present in the title, summary, introduction, methodology, results, and discussion of observational studies. This initiative aims to offer a recommended guideline on the most appropriate way to report observational studies, to facilitate critical reading by editors, reviewers, and readers in general. The checklist, however, should not be used to measure the quality of the studies being analyzed. ${ }^{27}$

The following strategy was used to complete the checklist and perform an analysis of each article: full compliance with the topic, so that all information regarding a specific item was present in the article; partial filling in of the topic, when the article contained some points required by the item, but not all; and non-compliance with the topic, which is the absence of any information required by that item.
The purpose of the GRADE system is to classify the quality of the evidence and the strength of the recommendations, taking into account the study design, the quality, consistency, and objectivity of the results, and the probability of bias. According to the GRADE system, the levels of evidence $\operatorname{are}^{28,29}$;

- High (A): Consistent evidence from randomized clinical trials with no significant limitations or exceptionally strong evidence from observational studies. Further research is unlikely to change the reliability of the effect estimate.

- Moderate (B): Evidence from randomized controlled trials with important limitations (such as inconsistent, indirect, or inaccurate results and flaws in the methodology) or very strong evidence from observational studies. Additional research is likely to have an important impact on the reliability of the effect estimate and may change it.

- Low (C): Evidence of at least one critical result from observational studies, case series, or randomized clinical trials, with serious flaws or indirect evidence. Additional research is very likely to have a major impact on the reliability of the effect estimate and is likely to change it.

- Very low (D): Any effect estimate is very uncertain.

\section{Results}

Of the 437 articles found, only 8 studies were selected for the review. The results of each stage of the study selection process, as well as the complete search strategy, can be seen in - Fig. 1.

Regarding the GRADE system, ${ }^{28}$ all studies were observational and classified in category $\mathrm{C}$, with a low level of evidence. - Table 1 shows the analysis of the articles, based on the STROBE initiative's checklist. ${ }^{27}$

Seven studies reported hearing loss in their patient populations and presented a diagnostic hypothesis of CQ or HCQinduced ototoxicity. ${ }^{3,5,6,13,17-19}$ One of the articles did not show conclusive evidence about hearing loss, since no significant hearing impairment was revealed in the group as a whole. Only 2 of the 11 participants showed changes in their results. ${ }^{15}$ Four studies classified the hearing losses as sensorineural, ${ }^{3,5,6,18}$ while three other publications did not include this information. ${ }^{13,17,19}$ The degree of hearing loss varied from mild to profound,,$^{3,5,17-19}$ and there was also diverse laterality. ${ }^{3,13,18}$

In six studies, CQ was administered, $5,13,15,17-19$ whereas, in two other reports, HCQ was prescribed. ${ }^{3,6}$ There was some variation in treatment doses among samples, mainly related to the age groups of the participants. In all eight studies, the patients underwent pure tone audiometry, $3,5,6,13,15,17-19$ while three studies also performed acoustic immittance testing. ${ }^{3,13,17}$ Only two investigations used brainstem evoked response audiometry (BERA). ${ }^{3,5}$

- Table 2 shows the data concerning the characteristics of the selected studies and exposure to CQ and HCQ. - Table 3 presents information about auditory symptoms and 


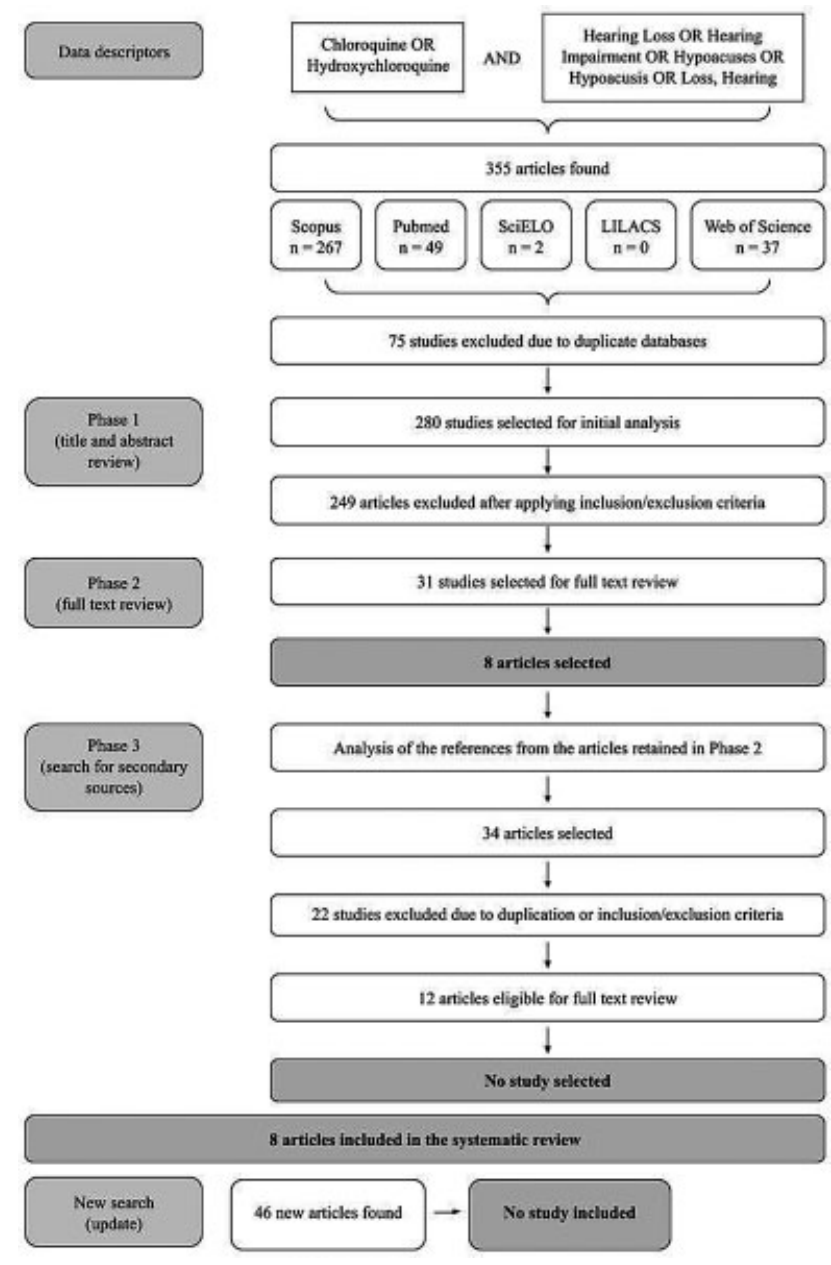

Fig. 1 Fluxogram.

methods of audiological testing and diagnoses. -Table 4 shows comparative data about exposure to the drugs, audiological outcomes, and reversibility of hearing loss.

\section{Discussion}

The present review aimed to investigate and analyze existing evidence in the literature about the toxic effects of $C Q$ and HCQ on the peripheral and/or central auditory system of its users. The data in the study selection showed that there is scientific evidence that these drugs can cause changes in the auditory system, such as hearing loss, mostly of the sensorineural type and with varying degrees. $3,5,6,13,17-19$

In the sample of selected articles, there was a wide variety among participants regarding age and gender. Most studies included children ${ }^{3,5,17-19}$ and non-elderly adults, ${ }^{6,13,15,17,19}$ but there was one article that also included elderly patients. ${ }^{17}$ As for gender, two articles reported on femaleonly samples ${ }^{3,18}$ and three populations only included males, $5,13,15$ while three other patient samples were mixed. ${ }^{6,17,19}$ However, since four articles studied patients diagnosed with malaria, $5,13,18,19$ there was not much variability concerning the diseases of the participants in the selected studies. The other articles presented pathologies
Table 1 Article analysis based on the STROBE initiative checklist

\begin{tabular}{|l|l|l|l|}
\hline Item & $\begin{array}{l}\text { Total } \\
\mathbf{n}(\%)\end{array}$ & $\begin{array}{l}\text { Partial } \\
\mathbf{n}(\%)\end{array}$ & $\begin{array}{l}\text { No } \\
\mathbf{n}(\%)\end{array}$ \\
\hline Title and abstract & $1(12.5 \%)$ & $7(87.5 \%)$ & $0(0 \%)$ \\
\hline Background/Rationale & $8(100 \%)$ & $0(0 \%)$ & $0(0 \%)$ \\
\hline Objectives & $2(25 \%)$ & $6(75 \%)$ & $0(0 \%)$ \\
\hline Study design & $7(87.5 \%)$ & $1(12.5 \%)$ & $0(0 \%)$ \\
\hline Setting & $6(75 \%)$ & $2(25 \%)$ & $0(0 \%)$ \\
\hline Variables & $4(50 \%)$ & $4(50 \%)$ & $0(0 \%)$ \\
\hline $\begin{array}{l}\text { Data sources/ } \\
\text { Measurement }\end{array}$ & $2(25 \%)$ & $4(50 \%)$ & $2(25 \%)$ \\
\hline Descriptive data & $0(0 \%)$ & $8(100 \%)$ & $0(0 \%)$ \\
\hline Main results & $4(50 \%)$ & $0(0 \%)$ & $4(50 \%)$ \\
\hline Limitations & $1(12.5 \%)$ & $2(25 \%)$ & $5(62.5 \%)$ \\
\hline Interpretation & $4(50 \%)$ & $3(37.5 \%)$ & $1(12.5 \%)$ \\
\hline Generalisability & $5(62.5 \%)$ & $1(12.5 \%)$ & $2(25 \%)$ \\
\hline
\end{tabular}

such as idiopathic pulmonary hemosiderosis, ${ }^{3}$ connective tissue disease, and subacute cutaneous lupus erythematosus. ${ }^{6}$ Chloroquine and HCQ are not well-known for treating the first two of these three diseases. In one of the articles, ${ }^{15}$ the participants were healthy (i.e., not affected by underlying diseases) and, in another study, ${ }^{17}$ the underlying condition was not mentioned. In all studies whose populations had malaria, CQ was prescribed to the patients..$^{5,13,18,19}$ However, no evidence was found to prove that HCQ is less effective than $\mathrm{CQ}$ in combating this disease.

As for the use of CQ and HCQ in research, it is known that both drugs show similar efficiency under diverse clinical conditions. ${ }^{30}$ Hydroxychloroquine is presented as less toxic than $\mathrm{CQ}^{4,8,9}$ and was considered a safer option in $1955 .^{30}$ However, HCQ was only used in two of the studies included in this review. ${ }^{3,6}$ In the other six articles, CQ was chosen for treatment ${ }^{5,13,15,17-19}$ and four of these studies were performed in developing countries, such as Nigeria ${ }^{17-19}$ and India. ${ }^{13}$ It is believed that this choice may be partly related to the antimalarial efficacy of CQ against erythrocyte invasion by Plasmodium falciparum parasites, as well as the cost and availability of this prescribed drug in these countries.

In two studies, other therapeutic methods were prescribed (medicated or not) in conjunction with CQ and HCQ. Coutinho and Duarte ${ }^{3}$ reported that the patient was prescribed the simultaneous use of HCQ and prednisolone. No associations were found between prednisolone and hearing loss. On the contrary, this drug was used to treat some cases of hearing loss. ${ }^{7,11}$ On the other hand, in the research of Kokong et al., ${ }^{17}$ they divided a sample of 156 patients into groups according to the medications that were administered, but only analyzed the data from a group of 22 participants who underwent CQ monotherapy.

Concerning the doses of CQ and HCQ prescribed in the research, the diversity among studies seems to be more 


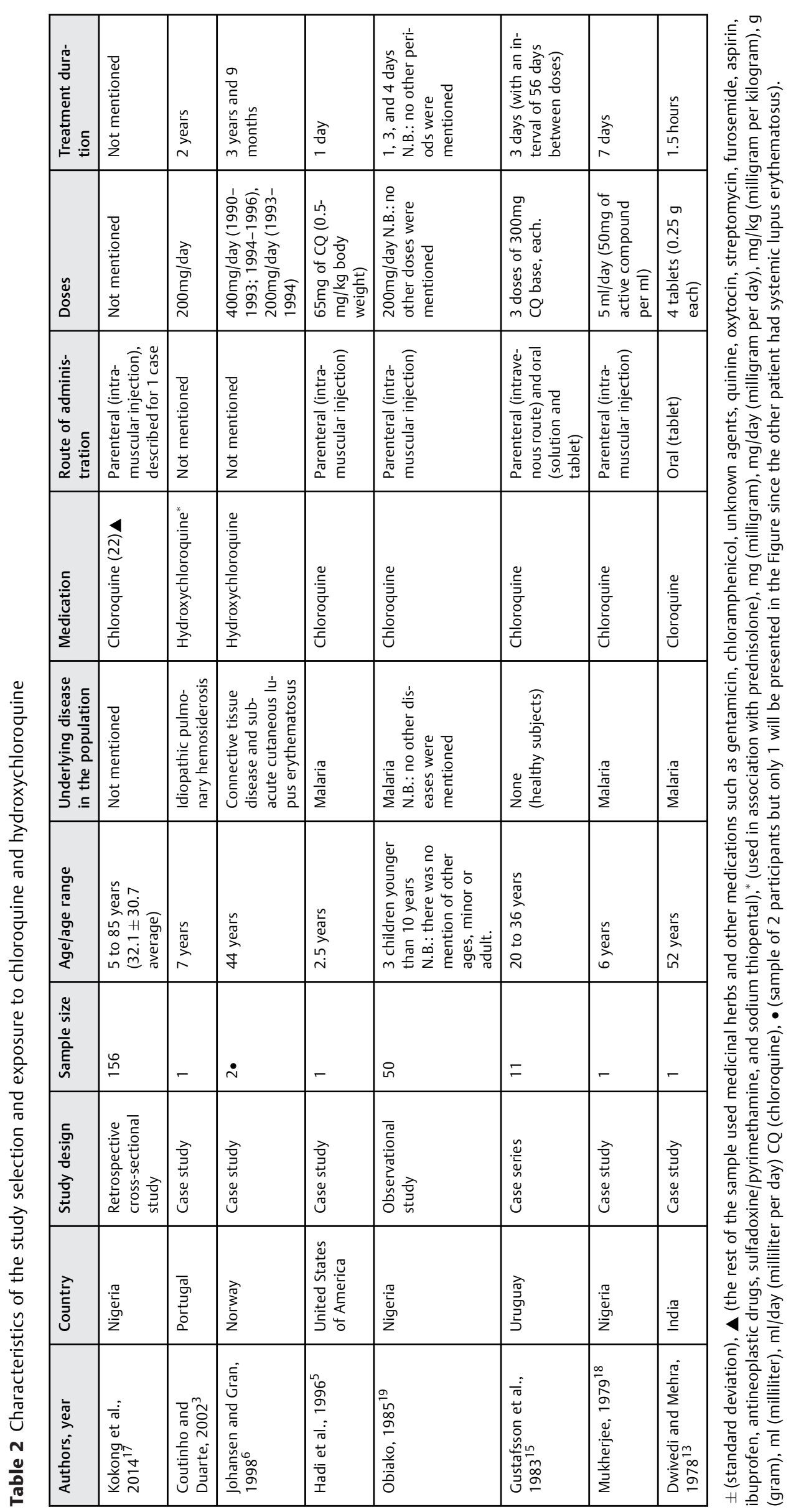




\begin{tabular}{|c|c|c|c|c|c|c|c|}
\hline 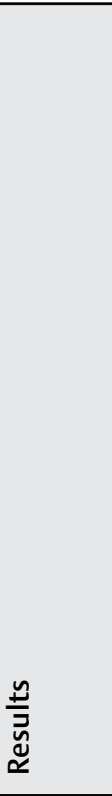 & 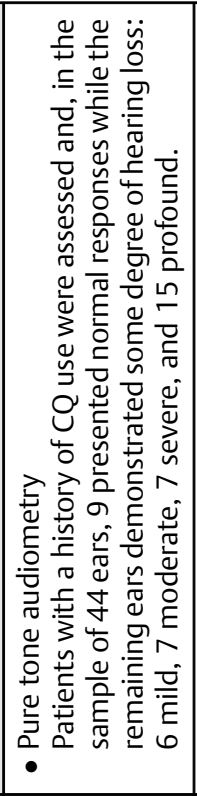 & 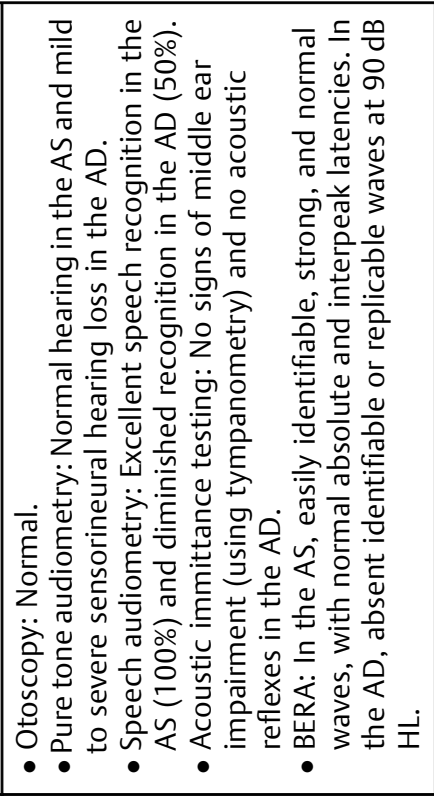 & 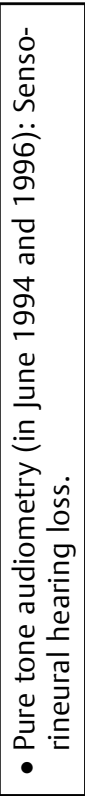 & 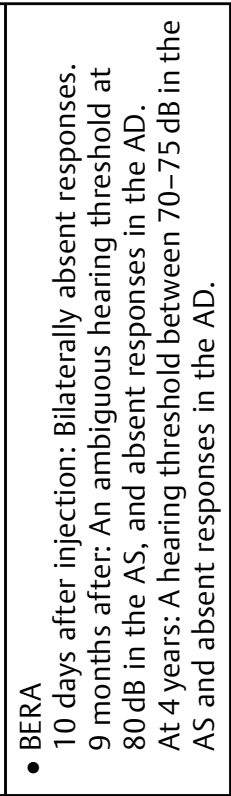 & 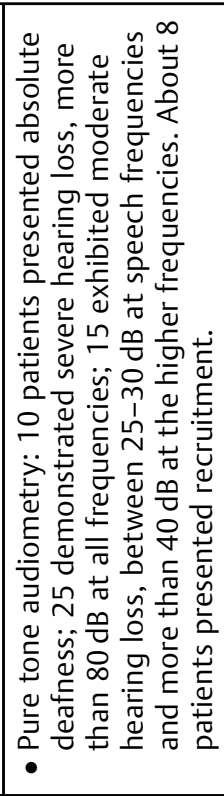 & 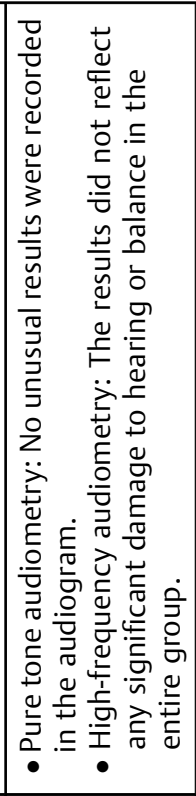 & 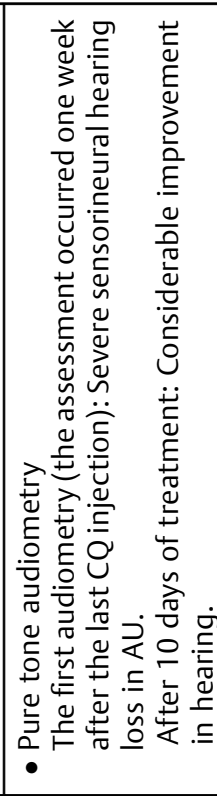 \\
\hline 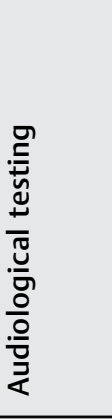 & 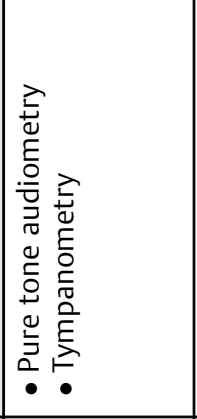 & 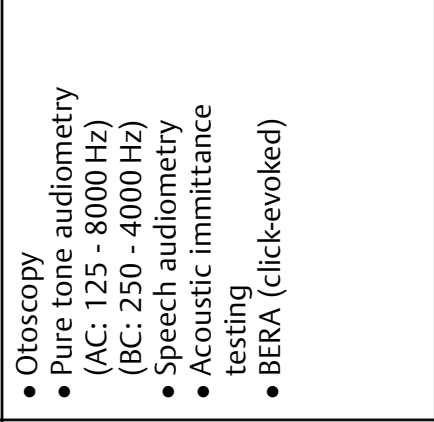 & 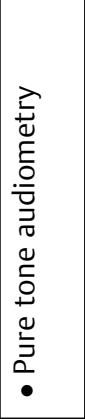 & 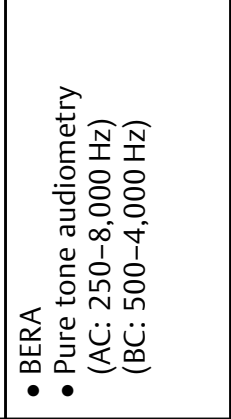 & 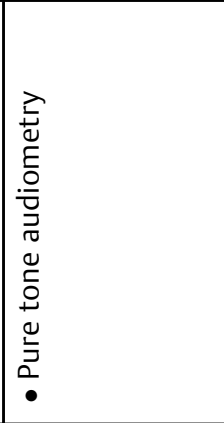 & 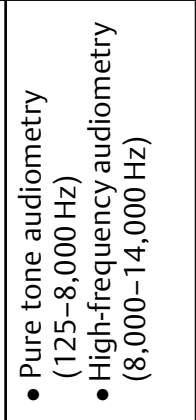 & 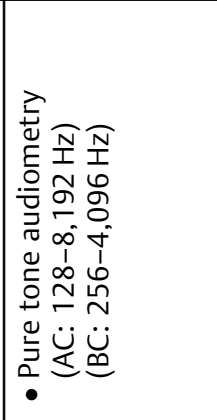 \\
\hline 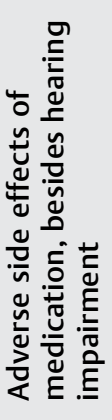 & 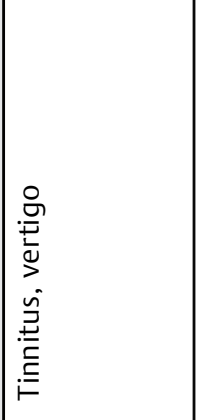 & 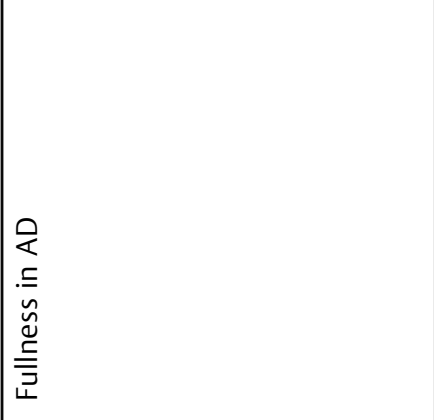 & 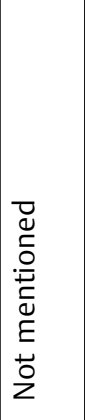 & 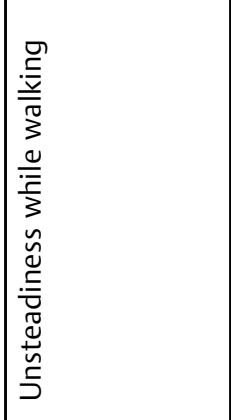 & 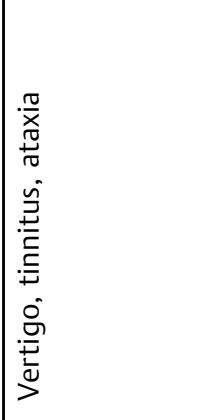 & $\mid \begin{array}{l}\widetilde{\widetilde{u}} \\
\stackrel{\bar{N}}{N} \\
\bar{\Delta}\end{array}$ & 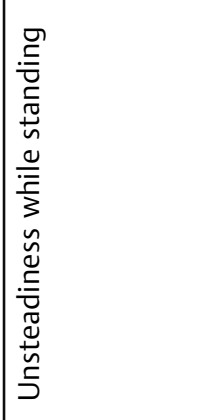 \\
\hline 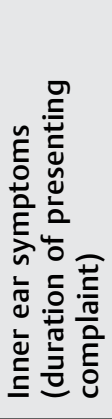 & 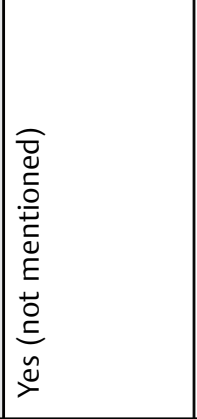 & 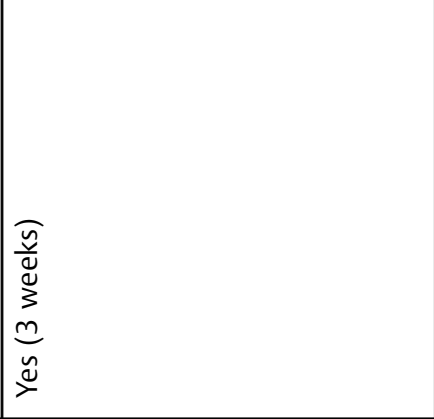 & 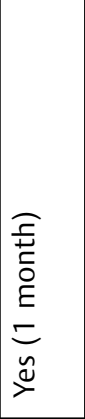 & 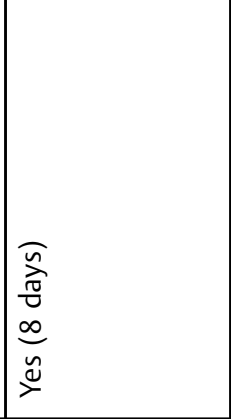 & 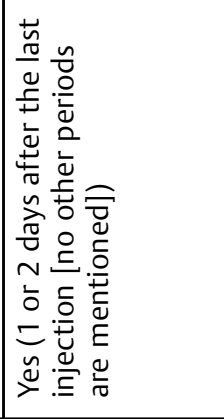 & z & 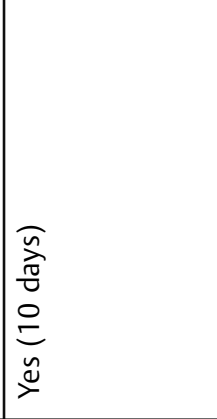 \\
\hline 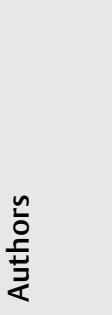 & 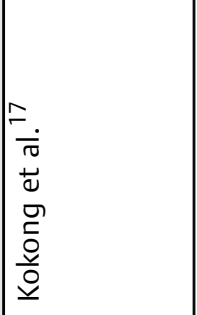 & 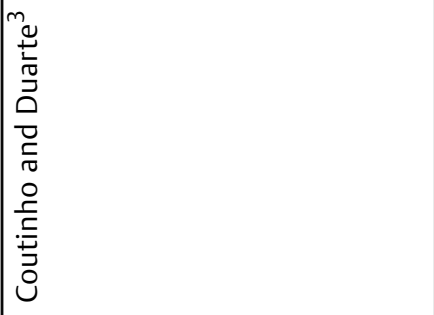 & 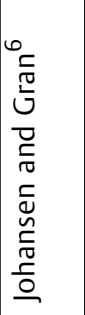 & 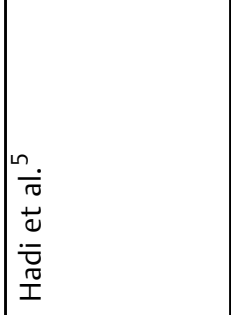 & $\frac{0}{\frac{0}{\sqrt[T]{L}}}$ & 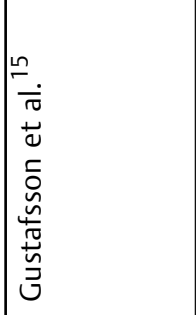 & 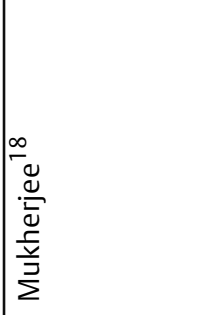 \\
\hline
\end{tabular}




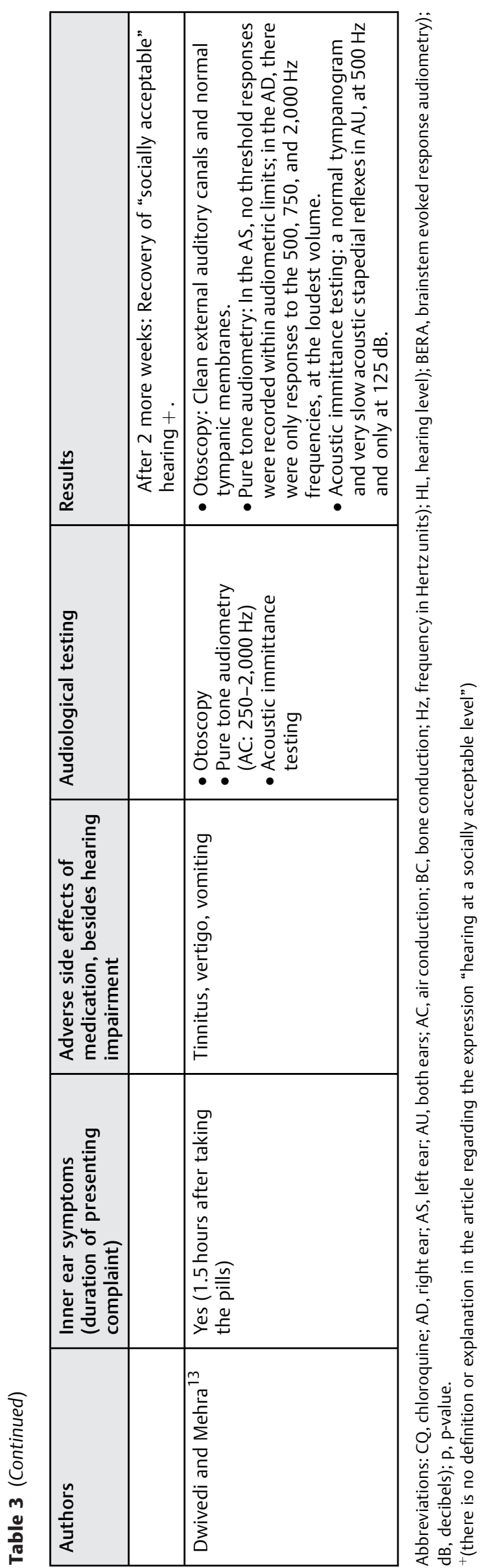

related to the age of patients rather than their diseases. Two of the lowest doses found in the study selection were prescribed for children. ${ }^{5,18}$ In the four articles that addressed patients with malaria, $5,13,18,19$ each one described a different dosage. Treatment duration also varied, depending on how chronic the disease was. Individuals with malaria ${ }^{5,13,18,19}$ were all treated for less than 10 days. In two other studies, 3,6 during which idiopathic pulmonary hemosiderosis, connective tissue disease, and subacute cutaneous lupus erythematosus were treated, the duration was much longer (more than 2 years).

During the selection of articles for this review, we excluded all articles in which hearing loss in the population could have occurred due to the complications of a disease, or the progression of an underlying condition.

Regarding the conditions of the patients that composed the samples of the selected studies (e.g., malaria, idiopathic pulmonary hemosiderosis, subacute cutaneous lupus erythematosus, and connective tissue disease), none of the compiled reports demonstrated evidence confirming the impact of any of these pathologies on the peripheral and/or central auditory system. As such, it was possible to proceed with our investigation of the ototoxic properties of medication and the effects of exposure.

As for monitoring ototoxicity, according to the American Academy of Audiology, ${ }^{31}$ assessment is more clearly defined when it comes to testing the peripheral hearing function. However, there is still a need for a standard protocol for clinical practice. The tests that have been used most recently are conventional pure tone audiometry, high-frequency audiometry, and otoacoustic emissions. ${ }^{31}$ However, only using conventional audiometry makes it impossible to detect ototoxic changes early. For this reason, high-frequency audiometry and otoacoustic emissions are more suitable tests since they allow professionals to identify significant auditory changes earlier than with pure tone audiometry. ${ }^{31}$

Ideally, an audiological assessment should be performed before any pharmacological prescription is issued. The main responsibility in monitoring for toxicity lies in the comparison of audiological tests. This makes it possible to detect any changes in results. The battery of tests should include pure tone audiometry, high-frequency audiometry, tympanometry, speech audiometry, and otoacoustic emissions. ${ }^{31}$

Bernard ${ }^{1}$ investigated the early detection of ototoxic changes by researching patients exposed to CQ. Using pure tone audiometry, the researcher noted that, although the auditory thresholds of the population remained unchanged, there were abnormal BERA results for 13 patients. The absolute latencies of waves III and V increased, while the latency of wave I remained stable. The I-III and I-V interpeak latencies also increased, but changes in the III-V interpeak latency were not as significant. The study demonstrated that the first signs of ototoxicity can be detected through BERA testing. In the selected studies in this review, these questions were not addressed, suggesting that further and more precise research is needed to support hypotheses about the neural injury that may be caused by these two drugs. 


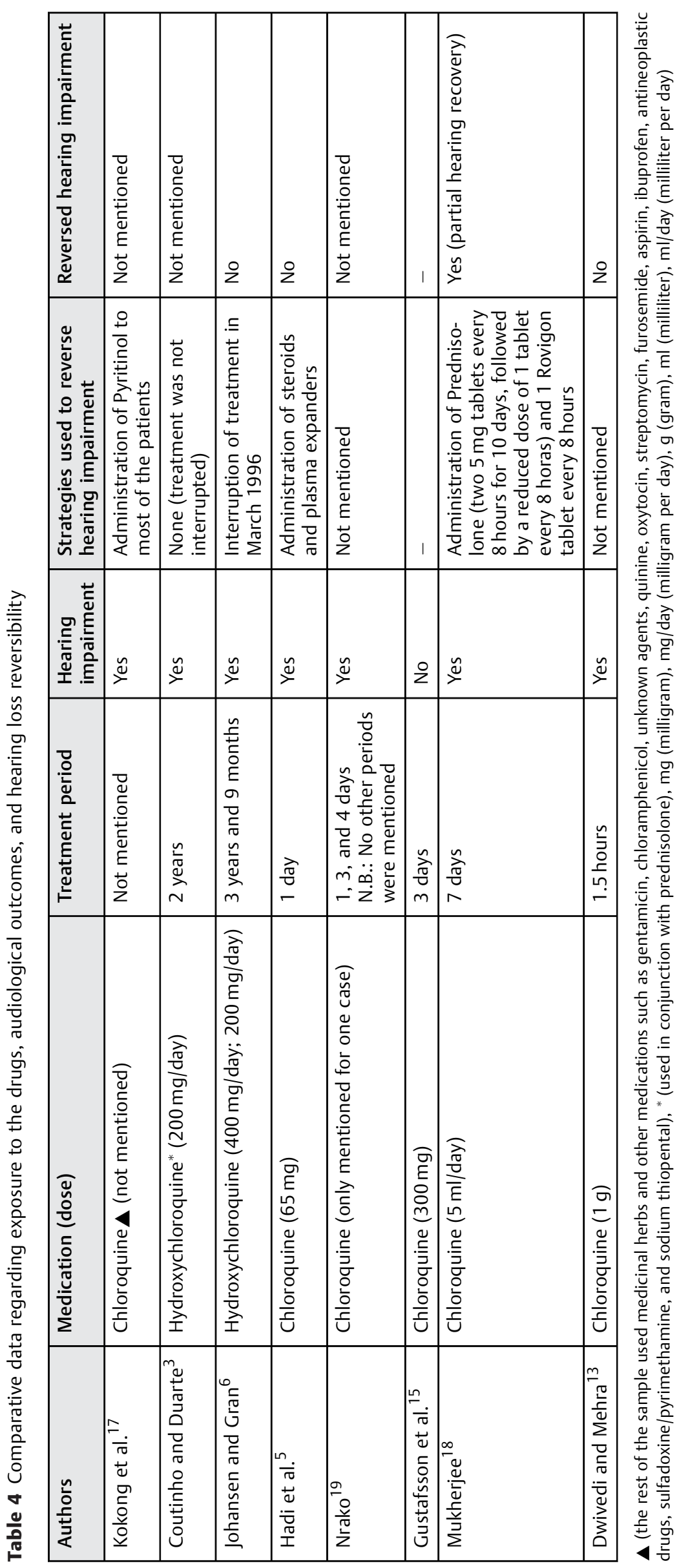


The audiological evaluation and tests presented in the articles included: pure tone audiometry, ${ }^{3,5,6,13,15,17-19}$ high frequency audiometry, ${ }^{15}$ speech audiometry, ${ }^{3}$ acoustic immittance testing ${ }^{3,13,17}$ and BERA. ${ }^{3,5}$ Half of the articles in this review used only behavioral hearing tests, ${ }^{6,15,18,19}$ while the other half used both behavioral and objective tests. $3,5,13,17$ Only two studies used electrophysiological tests. ${ }^{3,5}$ Pure tone audiometry was the most frequently performed assessment and was often the only examination that the patient underwent. ${ }^{6,18,19}$ High-frequency audiometry and speech audiometry were the least used exams. Coutinho and Duarte ${ }^{3}$ presented the most complete battery of audiological testing, per the recommendations of the American Academy of Audiology ${ }^{31}$ for monitoring ototoxicity.

Regarding audiological results, seven studies reported hearing loss among the participants in their samples. $^{3,5,6,13,17-19}$ Sensorineural hearing loss was prevalent in all studies that described the type of hearing loss. $3,5,6,18$ This fact may be associated with cochlear damage these two drugs cause since, in cases of hearing losses induced by ototoxicity, the damage is usually exclusively cochlear. ${ }^{31}$ The degree of hearing loss also varied considerably between reports. Besides this, the researchers did not identify the classifications they used to describe the patients' hearing loss. This makes it even more difficult to understand the real effect of CQ and HCQ in the IE. Only three articles described the laterality of the hearing loss $3,13,18$ after using pure tone audiometry. In two of them, ${ }^{13,18}$ the losses occurred in both ears, and, in another article, ${ }^{3}$ the impairment was one-sided.

In the two studies that performed BERA testing, ${ }^{3,5}$ there was no response in the right ear. However, waves were present for the left ear (with normal absolute and interpeak latencies in one study, ${ }^{3}$ and altered electrophysiological thresholds in the other ${ }^{5}$ ). There is no known scientific evidence to justify or clarify the worse performances of the right ear in audiological tests, but such results have already been published by Cunha et al. $^{32}$ based on research performed on Wistar rats exposed to toxic agents. The rats presented worse distortion product otoacoustic emissions (DPOAEs) results in the right ear.

The BERA results from the two articles mentioned above $^{3,5}$ reinforce the hypothesis that CQ and HCQ end up exerting a damaging influence on the IE, and probably sensory hair cells. The BERA findings of both studies showed that, although there were changes, they corroborated the pure tone audiometry results that had already shown characteristics reflecting cochlear impairment. When electrophysiological thresholds increase, the absolute and interpeak latencies of waves I, III, and V stay within normal range, and the interaural time difference is less than 0.3 milliseconds, so it is likely that the impairment was originated in the cochlea. ${ }^{33}$

The selected literature did not reveal any standard audiometric configuration or an audiological profile for cases of hearing impairment induced by CQ or HCQ. However, some articles ${ }^{3,34}$ define the hearing loss caused by quinine (a drug with a similar molecular structure and antimalarial action). ${ }^{18}$ Quinine-induced hearing impairment is generally of the sensorineural type, with mild-to-moderate, bilateral, and symmetrical losses. Quinine ototoxicity produces a hearing loss curve that is, in most cases, flat, or evident only at high frequencies. ${ }^{3,34}$

Concerning the reversibility of hearing loss, only one article $^{18}$ describes an improvement in hearing, although the recovery was not complete. In three studies, $5,6,13$ hearing loss was permanent and, in another three, ${ }^{3,17,19}$ this information was not included. The lesions caused by the action of ototoxic drugs are, for the most part, irreversible and can lead to the progressive damage of cochlea hair cells and changes in the stria vascularis. These changes alter the composition of endolymph. ${ }^{35}$ Interrupting treatment can lead to the suspension of adverse transformations in the endolymph, which can give reversible characteristics to any lesions that may be present. ${ }^{35}$ Failure to recover healthy hearing thresholds, even after discontinuing treatment, may be related to the loss of OHCs or IHCs. In the case of the article that reported an improvement in hearing, ${ }^{18}$ while the study did not mention if any prescribed medication had been suspended, the authors did describe the use of corticosteroids to attempt to reverse hearing loss. According to Mukherjee, ${ }^{18}$ corticosteroids may have controlled inflammatory reactions in the arteries or the hypersensitive reaction of the cochlear arteries to the $C Q$, allowing normal blood supply to be restored and cochlear responses to improve. These changes resulted in the partial recovery of hearing thresholds.

Regarding the origin of the ototoxicity of these drugs, there are some hypotheses about the etiology of CQ ototoxicity. All of them, in some way, relate to findings of the drug's affinity with melanocytes. According to Savin, ${ }^{36}$ the presence of melanocytes in the IE has been extensively studied over the years. These cells are present in the spiral ligament, stria vascularis (in greater quantity), modiolus, bony spiral lamina, and planum semilunatum. These structures are richly vascularized, and melanocytes tend to adhere directly to the walls of the vessels or regions closest to them. Lindquist and Ullberg $^{24}$ were the first researchers to establish a relationship between the changes caused by CQ in the IE and the affinity of the drug for melanin. In their study, large concentrations of the drug were found in the IEs of the fetuses of pigmented rats in their final stages of development. This correlation was reinforced when Dencker and Lindquist ${ }^{23}$ observed an intense accumulation of CQ in the stria vascularis, modioli, plana semilunata, saccule and utricle walls, and semicircular ducts of pigmented rats. However, these animals presented no drug accumulation in the endolymph, perilymph, sensory cells, or nerves.

Some authors ${ }^{5,18}$ believe that the auditory alterations could be caused by cochlear artery spasms, due to the sensitivity of these vessels to CQ. These contractions would, consequently, interfere with the oxygen supply to the sensory hair cells and stria vascularis. Hadi et $\mathrm{al}^{5}$ assume that this ischemia can lead to varied lesions in the cochlear hair cells, a reduction in the number of neurons, loss of support cells, and atrophy of the stria vascularis. Furthermore, Dencker and Lindquist ${ }^{23}$ suggest that the process could be 
somewhat more indirect. The stria vascularis and as the planum semilunatum are structures that produce endolymph, a fluid that bathes the apical regions of hair cells (ciliary tips). ${ }^{23,36}$ Vascular lesions or degenerative processes in these structures, caused by an accumulation of CQ in melanocytes, can lead to changes in the composition of the endolymph and secondary damage to sensory cells. ${ }^{23}$

The exact mechanism induced by HCQ in the IE is not fully understood. All articles that try to explain its effects ${ }^{7,11,14,37}$ mention existing hypotheses about the impact of CQ (basically, cochlear damage caused by ischemia). Because these drugs have similar structures and derive from the same compound, it is reasonable to assume that there may be similar ototoxic properties. Therefore, it is assumed that CQ and HCQ can affect the peripheral auditory system; more specifically, the cochlea.

Concerning the associations between pharmacological factors and audiological consequences, attempts were made to establish a relationship between the route of administration, dosage, or treatment duration with the severity of the hearing impairment presented by the research population. It should be noted that the rate of absorption of a drug depends, to a certain degree, on its route of administration. Oral absorption rates vary and depend on a few factors, but there may be a decrease in absorption due to the characteristics of the medication. In general, intramuscular administration leads to faster absorption than oral administration, especially with aqueous solutions. ${ }^{38}$ However, no differences were found among the articles regarding the route of administration and the degree of hearing impairment. In general, severe and profound hearing losses occurred in studies administering the drugs orally $^{13}$ and in those that used the intramuscular route. ${ }^{5,17-19}$

Similarly, no association was established between dosages and degrees of hearing loss. In the two articles in which two children were given doses of less than $200 \mathrm{mg}$ per day, the authors reported profound ${ }^{5}$ and severe ${ }^{18}$ hearing loss; the same severity reported by articles that used much higher doses. Additionally, no association was established between treatment duration and the degree of hearing loss. Only two articles ${ }^{3,6}$ described longer treatment times, and in one, ${ }^{6}$ the degree of hearing loss is not even mentioned.

All selected studies were observational, and there was a low level of evidence. According to the GRADE system, ${ }^{28}$ all selected articles fall into category $C$ and are classified as low evidence documents. However, in general, the studies followed the checklist items of the STROBE initiative, ${ }^{27}$ in whole or in part. There was inconsistency in the information presented in one article $^{15}$ regarding design. Studies must adequately describe the design of their work for a proper understanding of methodological procedures. Four articles presented their variables clearly and completely, $3,6,13,15$ while the other four described them partially. $5,17-19$

Most of the publications included in this review are case reports or case series, $3,5,6,13,15,18$ presenting inconsistent and under-structured methodologies. In general, the sample sizes are smaller, and more information is needed, especially in the descriptions of the results. Overall, there is a need for more detailed information and appropriate design choices so that better evidence can be produced.

A pattern of incomplete audiological information also stands out, especially in the description of assessments (e.g., equipment, type of stimuli, and method of performance) and the results of these tests (e.g., characterizations of the type, degree, and laterality of hearing loss). Only one article $^{17}$ presented a system for classifying the degrees of severity, based on the type of hearing loss. The rest did not describe how the averages were calculated or explain the severity of hearing losses. Four studies presented auditory imaging tests. 3,5,13,18 However, when they were obtained, only pure tone audiometry and BERA imaging were shown. These audiograms were not described or explored in the texts, leaving the interpretation of the exams up to the reader.

The number of selected articles in this review was small. This probably occurred due to the exclusion criterion about hearing loss caused by diseases (SLE and RA mainly). This led to the exclusion of many studies, since most research that describes CQ or HCQ-induced ototoxicity includes patients with these underlying conditions in population samples. In the same manner, the difficulty in establishing greater associations between the degree of hearing impairment and other issues, such as dosage and route of administration, may also be due to the small sample sizes in the selected research.

Another point to be highlighted is the fact that none of the studies performed audiological assessments before issuing pharmacological prescriptions. All tests were performed only after using the drugs and receiving hearing loss complaints. Therefore, there were no previous results with which a comparison could be made, as the American Academy of Audiology recommends. ${ }^{31}$

Since CQ and HCQ are effective drugs in the treatment of diseases such as malaria and SLE (in their different presentations) and are being investigated as potentially useful drugs in the treatment of other conditions, it is evident that we need studies with adequate designs that aim to further identify CQ and HCQ ototoxic mechanisms.

\section{Final Comments}

The present literature review led us to conclude that CQ and HCQ are capable of inducing hearing impairment in their users. The most common finding was sensorineural hearing loss, most likely due to cochlear damage. No differences were found regarding the ototoxic properties of the two drugs. Further studies with greater methodological rigor are needed for the continued clarification of the subject.

\section{Declarations}

No funding was received for conducting this study. This study did not involve human or animal subjects. All data generated or analyzed during this study are included in this published article. 


\section{Conflict of Interests}

The authors have no conflict of interests to declare.

\section{References}

1 Bernard P. Alterations of auditory evoked potentials during the course of chloroquine treatment. Acta Otolaryngol 1985;99(34):387-392

2 Chowdhury MS, Rathod J, Gernsheimer J. A Rapid Systematic Review of Clinical Trials Utilizing Chloroquine and Hydroxychloroquine as a Treatment for COVID-19. Acad Emerg Med 2020;27 (06):493-504

3 Coutinho MB, Duarte I. Hydroxychloroquine ototoxicity in a child with idiopathic pulmonary haemosiderosis. Int J Pediatr Otorhinolaryngol 2002;62(01):53-57

4 Figueiredo MC, Atherino CCCT, Monteiro CV, Levy RA. Antimalarials and Ototoxicity. Rev Bras Reumatol 2004;44(03):212-214

5 Hadi U, Nuwayhid N, Hasbini AS. Chloroquine ototoxicity: an idiosyncratic phenomenon. Otolaryngol Head Neck Surg 1996; 114(03):491-493

6 Johansen PB, Gran JT. Ototoxicity due to hydroxychloroquine: report of two cases. Clin Exp Rheumatol 1998;16(04):472-474

7 Patil A, Jerang Y, Mathew J. Hydroxychloroquine-induced auditory toxicity. Indian J Rheumatol 2020;15(01):53-55

8 Rynes RI. Antimalarial drugs in the treatment of rheumatological diseases. Br J Rheumatol 1997;36(07):799-805

9 Chen Z, Hu J, Zhang Z, et al. Efficacy of hydroxychloroquine in patients with COVID-19: results of a randomized clinical trial. MedRxiv 2020

10 Huang M, Tang T, Pang P, et al. Treating COVID-19 with Chloroquine. J Mol Cell Biol 2020;12(04):322-325

11 Khalili H, Dastan F, Dehghan Manshadi SA. A case report of hearing loss post use of hydroxychloroquine in a HIV-infected patient. Daru 2014;22(01):20

12 Rainsford KD, Parke AL, Clifford-Rashotte M, Kean WF. Therapy and pharmacological properties of hydroxychloroquine and chloroquine in treatment of systemic lupus erythematosus, rheumatoid arthritis and related diseases. Inflammopharmacology 2015; 23(05):231-269

13 Dwivedi GS, Mehra YN. Ototoxicity of chloroquine phosphate. A case report. J Laryngol Otol 1978;92(08):701-703

14 Seçkin U, Özoran K, Ikinciogullari A, Borman P, Bostan EE. Hydroxychloroquine ototoxicity in a patient with rheumatoid arthritis. Rheumatol Int 2000;19(05):203-204

15 Gustafsson LL, Walker O, Alván G, et al. Disposition of chloroquine in man after single intravenous and oral doses. Br J Clin Pharmacol 1983;15(04):471-479

16 Hart CW, Naunton RF. The Ototoxicity of Chloroquine Phosphate. Arch Otolaryngol 1964;80:407-412

17 Kokong DD, Bakari A, Ahmad BM. Ototoxicity in Nigeria: why it persists. Ear Nose Throat J 2014;93(07):256-264

18 Mukherjee DK. Chloroquine ototoxicity-a reversible phenomenon? J Laryngol Otol 1979;93(08):809-815

19 Obiako MN. Chloroquine ototoxicity: an iatrogenic problem. Mater Med Pol 1985;17(03):195-197

20 Toone EC, Hayden GD, Ellman HM. Ototoxicity of chloroquine. [abstract]Arthritis and Rheumatism (N.Y.) 1965;8(03):475-476

21 Chatelet JN, Auffret M, Combret S, Bondon-Guitton E, Lambert M, Gautier S. [Hydroxychloroquine-induced hearing loss:
First case of positive rechallenge and analysis of the French pharmacovigilance database]. Rev Med Interne 2017;38(05): 340-343

22 Lim SC, Tang SP. Hydroxychloroquine-induced ototoxicity in a child with systemic lupus erythematosus. [letter]Int J Rheum Dis 2011;14(01):e1-e2

23 Dencker L, Lindquist NG. Distribution of labeled chloroquine in the inner ear. Arch Otolaryngol 1975;101(03):185-188

24 Lindquist NG, Ullberg S. The melanin affinity of chloroquine and chlorpromazine studied by whole body autoradiography. Acta Pharmacol Toxicol (Copenh) 1972;2(Suppl):2, 1-32

25 Basic Ear and Hearing Care Resource [internet]. Geneva: World Health Organization; 2020. Licence: CC BY-NC-SA 3.0 IGO. Accessed Sept 28, 2020

26 Moher D, Liberati A, Tetzlaff J, Altman DGPRISMA Group. Preferred reporting items for systematic reviews and meta-analyses: the PRISMA statement. Ann Intern Med 2009;151(04):264-269, W64

27 Malta M, Cardoso LO, Bastos FI, Magnanini MMF, Silva CM. STROBE initiative: guidelines on reporting observational studies. Rev Saude Publica 2010;44(03):559-565

28 Atkins D, Best D, Briss PA, et al; GRADE Working Group. Grading quality of evidence and strength of recommendations. BMJ 2004; 328(7454):1490-1494

29 Guyatt GH, Norris SL, Schulman S, et al. Methodology for the development of antithrombotic therapy and prevention of thrombosis guidelines: Antithrombotic Therapy and Prevention of Thrombosis, 9th ed: American College of Chest Physicians Evidence-Based Clinical Practice Guidelines. Chest 2012;141(2, Suppl)53S-70S

30 Ben-Zvi I, Kivity S, Langevitz P, Shoenfeld Y. Hydroxychloroquine: from malaria to autoimmunity. Clin Rev Allergy Immunol 2012; 42(02):145-153

31 Position Statement and Clinical Practice Guidelines Ototoxicity Monitoring [internet]. American Academy of Audiology; 2009. Accessed Oct 05, 2020 at: https://audiology-web.s3.amazonaws.com/migrated/OtoMonGuidelines.pdf_539974c40999c1.58842217.pdf

32 Cunha EO, Reis AD, Macedo MB, Machado MS, Dallegrave E. Ototoxicity of cypermethrin in Wistar rats. Rev Bras Otorrinolaringol (Engl Ed) 2020;86(05):587-592

33 Andrade KCL, Pinheiro NS, Carnaúba ATL, Menezes PL. Potencial Evocado Auditivo de Tronco Encefálico: Conceitos e Aplicações Clínicas. In: Menezes PL, Andrade KCL, Frizzo ACF, Carnaúba ATL, Lins OG, (org). Tratado de Eletrofisiologia para a Audiologia. São Paulo: Book Toy; 2018:73-83

34 Scott PMJ, Griffiths MV. A clinical review of ototoxicity. Clin Otolaryngol Allied Sci 1994;19(01):3-8

35 Silveira SCR. Ototoxicidade. In: Piltcher OB, da Costa SS, Maahs GS, Kuhl G, (org) Rotinas em Otorrinolaringologia. Porto Alegre: Artmed; 2015:90-96

36 Savin C. The blood vessels and pigmentary cells of the inner ear. Ann Otol Rhinol Laryngol 1965;74(03):611-622

37 Fernandes MRN, Soares DBR, Thien CI, Carneiro S. Hydroxychloroquine ototoxicity in a patient with systemic lupus erythematosus. [letter]An Bras Dermatol 2018;93(03):469-470

38 Brum LFS, Rockenbach L, Bellicanta PL. Farmacocinética I - absorção de drogas. In: Brum LFS, ed. Farmacologia básica. Porto Alegre: SAGAH; 2018:15-35 